\title{
Prognostic effect and modulation of cardiac sympathetic function in heart failure patients treated with cardiac resynchronization therapy
}

\author{
Rita Ilhão Moreira, $M D,{ }^{a}$ Ana Abreu, $M D, P h D,{ }^{a}$ Guilherme Portugal, $M D,{ }^{a}$ \\ Luís Oliveira, $M D,{ }^{b}$ Mário Oliveira, $M D, P h D,{ }^{a}$ Inês Rodrigues, $M D,{ }^{a}$ \\ Madalena Coutinho Cruz, MD, ${ }^{a}$ Pedro Silva Cunha, MD, ${ }^{a}$ Vanessa Santos, SB, ${ }^{\mathrm{c}}$ \\ Helena Santa Clara, SB, PhD, ${ }^{\mathrm{c}}$ Miguel Mota Carmo, MD, PhD, ${ }^{\mathrm{a}, \mathrm{d}}$ and \\ Rui Cruz Ferreira, $M D^{a}$ \\ a Department of Cardiology, Hospital de Santa Marta, Centro Hospitalar de Lisboa Central, \\ Lisbon, Portugal \\ b Nuclear Medicine Department, Medical and Diagnosis Clinic Quadrantes, Lisbon, Portugal \\ c CIPER, Human Kinetics Faculty, University of Lisbon, Lisbon, Portugal \\ d NOVA Medical School, New University of Lisbon, Lisbon, Portugal
}

Received May 2, 2018; accepted Jun 11, 2018

doi:10.1007/s12350-018-1357-x

Background. Cardiac autonomic dysfunction as assessed by ${ }^{123}$ I-metaiodobenzylguanidine ( ${ }^{123} \mathrm{I}$-mIBG) scintigraphy is associated with poor prognosis in heart failure (HF) patients. Although cardiac resynchronization therapy (CRT) has emerged as an effective therapy in improving outcomes on HF patients, its effect on cardiac sympathetic nervous function is still not fully understood. We aimed to study the value of pre-implantation ${ }^{123}$ I-mIBG late heart-tomediastinum ratio (HMR) as a predictor of response and outcomes after CRT and to correlate modification in this parameter with CRT response and functional improvement.

Methods and Results. BETTER-HF (Benefit of exercise training therapy and cardiac resynchronization in $\mathrm{HF}$ patients) is a prospective randomized clinical trial including $\mathrm{HF}$ patients submitted CRT (mean LVEF $24 \pm 8 \%, 74 \%$ NYHA class $\geq$ III) who underwent a clinical, echocardiographic, and scintigraphic assessment before and 6 months after CRT. Onehundred and twenty-one patients were included. Echocardiographic response was observed in $54 \%$ and composite outcome of cardiac mortality, cardiac transplant or heart failure hospitalization in $24 \%$ of patients. Baseline late HMR was an independent predictor of CRT response (regression coefficient $2.906,95 \%$ CI $0.293-3.903, P$.029) and outcomes (HR 0.066 95\% CI 0.005-0.880, $P$.040). At follow-up, ${ }^{123}$ I-mIBG imaging showed positive changes in cardiac sympathetic nerve activity only in responders to CRT $(1.36 \pm 0.14$ prior vs. $1.42 \pm 0.16$ after CRT, $P$.039). There was a significant correlation between improvement in late HMR and improvement in peak oxygen consumption $(r$ 0.547, $P<.001)$.

Electronic supplementary material The online version of this article (https://doi.org/10.1007/s12350-018-1357-x) contains supplementary material, which is available to authorized users.

The authors of this article have provided a PowerPoint file, available for download at SpringerLink, which summarises the contents of the paper and is free for re-use at meetings and presentations. Search for the article DOI on SpringerLink.com.
Reprint requests: Rita Ilhão Moreira, MD, Department of Cardiology, Hospital de Santa Marta, Centro Hospitalar de Lisboa Central, Rua de Santa Marta, no. 50, 1169-024 Lisbon, Portugal; ritailhaomoreira@gmail.com

1071-3581/\$34.00

Copyright (C) 2018 American Society of Nuclear Cardiology. 
Conclusion. In our study, baseline cardiac denervation predicted response and clinical outcomes after CRT implantation. Cardiac sympathetic function was improved only in patients who responded to CRT and these positive changes were correlated with improvement in functional capacity. (J Nucl Cardiol 2020;27:283-90.)

Key Words: Heart failure $\cdot$ cardiac innervation $\cdot$ diagnostic and prognostic application

\begin{tabular}{|ll|}
\hline \multicolumn{2}{|l|}{ Abbreviations } \\
HF & Heart failure \\
${ }^{123}$ I-mIBG & ${ }^{123}$ I-metaiodobenzylguanidine \\
HMR & Heart-to-mediastinum ratio \\
CRT & Cardiac resynchronization therapy \\
LVEF & Left ventricular ejection fraction \\
LVESV & Left ventricle end-systolic volume \\
VO2 & Oxygen consumption \\
BNP & Brain natriuretic peptide \\
Hs-CRP & High-sensitive C-reactive protein \\
\hline
\end{tabular}

See related editorial, pp. 291-293

\section{INTRODUCTION}

Sympathetic activation is a key pathophysiological mechanism in chronic heart failure (HF) ${ }^{1,2}$ Specific cardiac sympathetic nerve activity can be assessed by ${ }^{123}$ I-metaiodobenzylguanidine $\quad\left({ }^{123} \mathrm{I}-\mathrm{mIBG}\right)$ scintigraphy, since it shares the same presynaptic uptake, storage, and release mechanisms as norepinephrine, but is biologically inactive. The semi-quantified parameters of ${ }^{123} \mathrm{I}$-mIBG myocardial uptake and washout, i.e., late heart-to-mediastinum ratio (HMR), were proven to be of prognostic value in HF patients. ${ }^{3,4}$

In the last two decades, cardiac resynchronization therapy (CRT) has been shown to improve left ventricular ejection fraction (LVEF), reduce heart failure hospitalizations, and decrease all-cause mortality in patients with $\mathrm{HF}$ and electrical dyssynchrony ${ }^{5-7}$ However, its effect on cardiac sympathetic nervous function is still not fully well known.

Our purpose was to investigate the value of baseline cardiac sympathetic activity, assessed by ${ }^{123}$ I-mIBG late HMR, as a predictor of response and outcomes in HF patients undergoing CRT implantation. Additionally, we sought to correlate changes in ${ }^{123} \mathrm{I}-\mathrm{mIBG}$ late HMR after device implantation with CRT response and functional improvement at 6-month follow-up.

\section{METHODS}

\section{Study Design}

BETTER-HF (NCT02413151) is a prospective cohort of consecutive patients with $\mathrm{HF}$ and reduced ejection fraction (HFrEF) submitted to cardiac resynchronization therapy (CRT) enrolled between April 2011 and February 2015 in a single centre. Heart failure patients in NYHA class III-IV with LV dysfunction (LVEF $<35 \%$ ), QRS duration wider than 120 millisecond, and dyssynchrony, referred for CRT implantation were included. Patients who refused participation and who did not sign the informed consent were excluded.

The protocol of the study has been detailed in a prior publication. ${ }^{8}$ Patients were subjected to a baseline assessment before CRT, including clinical evaluation, blood analysis, and transthoracic echocardiogram and were revaluated at 6 months after CRT. ${ }^{123}$ I-mIBG scintigraphy was performed in all patients at baseline. When feasible due to distance of resident and costs, patients were submitted to ${ }^{123} \mathrm{I}$-mIBG scintigraphy revaluation at 6 months after enrolment.

All patients signed a written informed consent form before the performance of any procedure. The study was approved by the Institutional Review Board and Ethics Committee.

\section{${ }^{123}$ I-MIBG CARDIAC SCINTIGRAPHY ACQUISITION AND PROCESSING}

${ }^{123}$ I-mIBG scintigraphy was performed by obtaining planar images in an anterior projection using a singleheaded Siemens e.Cam camera fitted with a low-energy high-resolution collimator. Counts were acquired for 240 seconds into a $128 \times 128$ pixel matrix 15 minutes (early) and 4 hours (late) after the intravenous injection of $185 \mathrm{MBq}$ of ${ }^{123} \mathrm{I}-\mathrm{mIBG}$. The main energy window was centered to $15 \%$ of the $159-\mathrm{keV} 123 \mathrm{I}$ photo peak. No scatter correction was applied. Thyroid blockade with potassium iodide was performed $>30$ minutes before ${ }^{123} \mathrm{I}-\mathrm{mIBG}$ injection. Cardiovascular drugs, namely beta-blockers, were maintained at both acquisitions. Antidepressants were interrupted, whenever possible. Images were processed on a WS Siemens Syngo workstation.

Early and late heart-to-mediastinum ratio (HMR) values were calculated after measuring the mean count of ${ }^{123} \mathrm{I}$-mIBG uptake per pixel in two separate visually drawn regions of interest: around the entire heart $(\mathrm{H})$ and over the midline upper mediastinum to reflect the location with lowest activity (M). The washout rate was expressed as a percentage and calculated according to this formula: ((Early M-early $\mathrm{H})$ - (late M-late $\mathrm{H})$ )/ (early M-early $\mathrm{H}) * 100$.

In order to confirm reproducibility of this imaging technique, all the scans were evaluated by two separate readers with the average value for each parameter taken for the main analysis.

Previous analysis of this population showed good inter-observer agreement of HMR, as measured by the 
intraclass correlation coefficient, indicating good to excellent reproducibility. ${ }^{8}$

\section{Echocardiographic Acquisition and Processing}

A GE Vivid 9 ultrasound system was used to acquire parasternal long- and short-axis views, as well as apical two-, three-, and four-chamber views. Echocardiographic parameters, including left ventricle enddiastolic and end-systolic volumes and left ventricle ejection fraction, were determined according to the American Society of Echocardiography's recommendations. ${ }^{9,10}$

\section{Definitions}

Echocardiographic response to CRT was defined as left ventricle end-systolic volume (LVESV) decrease $\geq 15 \%$. $^{11}$ Composite outcome was defined as cardiac mortality, cardiac transplant or heart failure hospitalization. Functional capacity was assessed with cardiopulmonary exercise test and peak oxygen consumption (VO2) was defined as the highest 30-second average value of VO2 achieved during exercise.

\section{Statistical Analysis}

Data were expressed as mean \pm standard deviation (SD) for continuous variables and as frequencies and percentages for categorical variables. Data distribution was tested for normality using the Kolmogorov-Smirnov test or Shapiro-Wilk as appropriate. Comparisons of baseline characteristics and response were performed using the Chi-square test or Fisher's exact test, when appropriate, for categorical variables, and the Student's $t$ test or the Mann-Whitney test for continuous variables. Logistic regression analysis was used to identify predictors of CRT response, and Cox proportional hazards regression analysis was performed to identify predictors of composite endpoint. The relationship between the change in late HMR and the change in other variables was examined by the determination of standardized correlation coefficients. All the statistical tests were twosided, and a $P$ value $<.05$ was considered significant. The SPSS version 21 software (SPSS Inc., Chicago, IL) was used for computation.

\section{RESULTS}

\section{Baseline Characteristics}

The study included 121 patients, average age of $67 \pm 11$ years, the majority of whom were men $(70.4 \%)$.
Baseline characteristics of the population are shown in Table 1. Most had non-ischemic cardiomyopathy $(72.2 \%)$, suffered from severe HF $(73.5 \%$ with NYHA class $\geq$ III), and had poor LV function (mean LVEF $24.2 \pm 6.7 \%)$.

\section{Baseline Cardiac Sympathetic Innervation and CRT Response}

Mean baseline late HMR was $1.38( \pm 0.18)$, early HMR 1.47 ( \pm 0.17$)$, and wash out rate 49\% ( \pm 18$)$.

CRT response ( $\geq 15 \%$ reduction in LVESV) was observed in $53.7 \%$ of patients at 6-month follow-up. There were no significant differences regarding age, gender, NYHA class nor comorbidities in the two groups (Table 1).

In a multivariable analysis adjusting for age, gender, aetiology, and left bundle branch morphology, late HMR was an important independent predictor of response to CRT (regression coefficient 2.906, 95\% CI 0.293-3.903, $P$.029).

\section{Baseline Cardiac Sympathetic Innervation and Long-Term Outcome}

During follow-up (mean $25.5 \pm 12.9$ months), composite endpoint was documented in 29 patients $(24.4 \%)$, corresponding to $11.5 \%$ per year.

Univariate analysis identified baseline estimated glomerular filtration rate, serum levels of brain natriuretic peptide (BNP) and high-sensitive C-reactive protein (hs-CRP), smoking habits, electrical dyssynchrony and late HMR as predictors of composite endpoint (Table 2). Multivariate Cox proportional hazards regression analysis using these significant predictors showed that late HMR was an independent powerful determinant of composite outcome (HR 0.066, 95\% CI 0.005-0.880, $P$.040).

\section{Cardiac Sympathetic Innervation Changes, CRT Response, and Functional Improvement}

Changes in different parameters induced by CRT are shown in Table 3. Significant improvement in NYHA class, LVEF, and LV end-systolic volume index were observed after CRT. ${ }^{123}$ I-mIBG parameters at 6month follow-up were available in 55 patients. When the entire cohort was analyzed, ${ }^{123}$ I-mIBG parameters did not show any significant change at follow-up when compared to baseline. However, when the groups were analyzed separately according to response to CRT, only echocardiographic responders showed a significant increase in late HMR at follow-up when compared to baseline $(1.36 \pm 0.14$ prior to $\mathrm{CRT}$ vs. $1.42 \pm 0.16$ six 
Table 1. Baseline characteristics according to CRT response

\begin{tabular}{|c|c|c|c|c|}
\hline Baseline characteristics & $\begin{array}{l}\text { All patients } \\
\qquad n=121\end{array}$ & $\begin{array}{l}\text { Responders } \\
n=65\end{array}$ & $\begin{array}{c}\text { Non-responders } \\
n=56\end{array}$ & $\boldsymbol{P}$ \\
\hline Age, years mean (SD) & $67( \pm 11)$ & $68( \pm 12)$ & $63( \pm 10)$ & .103 \\
\hline Male gender, n (\%) & $85(70)$ & $48(73)$ & $37(67)$ & .538 \\
\hline Ischaemic aetiology, n (\%) & $34(28)$ & $18(28)$ & $16(29)$ & .929 \\
\hline NYHA class $\geq$ III, n (\%) & $90(74)$ & $46(71)$ & 44 (79) & .581 \\
\hline Obesity, n (\%) & $34(28)$ & $20(31)$ & $14(25)$ & .609 \\
\hline Hypertension, n (\%) & $105(87)$ & $57(89)$ & $48(86)$ & .592 \\
\hline Dyslipidemia, n (\%) & $84(69)$ & $42(64)$ & $42(75)$ & .176 \\
\hline Diabetes, n (\%) & $46(38)$ & $23(35)$ & $23(41)$ & .495 \\
\hline History of smoking habits, n (\%) & $29(25)$ & 12 (19) & $17(30)$ & .198 \\
\hline Beta-blockers, n (\%) & $107(89)$ & $57(88)$ & $50(89)$ & .917 \\
\hline ACE-I or ARA, $n(\%)$ & $110(91)$ & $59(91)$ & $51(91)$ & .875 \\
\hline Left bundle branch block, n (\%) & $86(71)$ & 45 (69) & $41(73)$ & .566 \\
\hline QRS duration, ms mean (SD) & $145( \pm 24)$ & $145( \pm 24)$ & $142( \pm 20)$ & .48 \\
\hline $\mathrm{BNP}, \mathrm{pg} \cdot \mathrm{mL}^{-1}$ mean $(\mathrm{SD})$ & $490( \pm 404)$ & $515( \pm 481)$ & $480( \pm 312)$ & .132 \\
\hline hs-CRP, mg. $\mathrm{L}^{-1}$ mean (SD) & $3.2( \pm 2.8)$ & $2.0( \pm 0.9)$ & $4.4( \pm 4.0)$ & .73 \\
\hline eGFR, $\mathrm{mL} \cdot \mathrm{min}^{-1} / 1.73 \mathrm{~m}^{2}$ mean (SD) & $78( \pm 37)$ & $77( \pm 45)$ & $80( \pm 26)$ & .451 \\
\hline Peak VO2, $\mathrm{mL} \cdot \mathrm{kg}^{-1} \cdot \mathrm{min}^{-1}$ mean (SD) & $14.8( \pm 5.8)$ & $14.4( \pm 6.3)$ & $15.2( \pm 5.7)$ & .202 \\
\hline LVEF, \% mean (SD) & $24( \pm 7)$ & $24( \pm 7)$ & $24( \pm 9)$ & .065 \\
\hline LVEDV index, $\mathrm{mL} \cdot \mathrm{m}^{-2}$ mean (SD) & $127( \pm 40)$ & $129( \pm 73)$ & $122( \pm 38)$ & .448 \\
\hline LVESV index, $\mathrm{mL} \cdot \mathrm{m}^{-2}$ mean (SD) & $95( \pm 36)$ & $99( \pm 39)$ & $89( \pm 33)$ & .895 \\
\hline Early HMR & $1.47( \pm 0.17)$ & $1.48( \pm 0.17)$ & $1.46( \pm 0.18)$ & .202 \\
\hline Late HMR & $1.38( \pm 0.18)$ & $1.41( \pm 0.14)$ & $1.37( \pm 0.16)$ & .047 \\
\hline Wash out rate (\%) & $49( \pm 18)$ & $50( \pm 19)$ & $47( \pm 24)$ & .581 \\
\hline
\end{tabular}

NYHA, New York Heart Association; $A C E-I$, angiotensin-converting enzyme inhibitors; $A R A$, angiotensin II receptor blockers; $B N P$, brain natriuretic peptide; $h s-C P R$, high-sensitivity C-reactive protein; eGFR, estimated glomerular filtration rate; peak VO2, peak oxygen consumption; $L V E F$, left ventricular ejection fraction; $L V E D V$, LV end-diastolic volume; $L V E S V$, LV end-systolic volume; $H M R$, heart-to-mediastinum ratio

months after CRT, $P$.039), whereas non-responders did not show significant differences. There were no significant changes in early HMR nor washout rate.

Baseline clinical variables were not predictors of late HMR modifications.

Pearson's correlation analysis was performed to analyze the association between late HMR changes and modifications in clinical, laboratory, echocardiographic variables (Table 4). There was a moderate significant correlation between improvement in late HMR and improvement in hs-CRP $(r-0.356, P$.012 $)$ and in peak VO2 at 6 months $(r 0.547, P<.001$, Figure 1$)$.

\section{DISCUSSION}

The findings of the current study can be summarized as follows: (1) baseline late HMR could predict response and outcomes in HF patients undergoing CRT; (2) CRT responders improved cardiac sympathetic nerve dysfunction, whereas non-responders did not show any significant changes and (3) after CRT there was a significant correlation between improvement in late HMR and improvement in peak VO2.

\section{Baseline Cardiac Denervation, CRT Response, and Outcomes}

Adrenergic activation is recognized as an important factor in the pathogenesis and outcome of $\mathrm{HF}^{12,13} \mathrm{In}$ response to a deterioration of cardiac function, increased neuronal release of norepinephrine (NE) is accompanied by a decrease in presynaptic NE reuptake due to downregulation of the cardiac NE transporter. If continued, reactive desensitization of the myocardial betaadrenergic receptors in the synaptic cleft develops and cardiac pump failure is further exacerbated. Cardiac ${ }^{123}$ I-mIBG scintigraphy provides information on the 
Table 2. Univariate and multivariate analysis of predictors of composite endpoint

\begin{tabular}{|c|c|c|c|c|}
\hline \multirow[b]{2}{*}{ Dependent variable } & \multicolumn{2}{|c|}{ Univariable analysis } & \multicolumn{2}{|c|}{ Multivariable analysis } \\
\hline & HR $(95 \% \mathrm{CI})$ & $\boldsymbol{P}$ & HR (95\% CI) & $\boldsymbol{P}$ \\
\hline Age & $1.026(0.992-1.061)$ & .132 & - & \\
\hline Female gender & $0.297(0.151-1.042)$ & .061 & - & \\
\hline Obesity & $1.586(0.645-3.901)$ & .315 & - & \\
\hline Diabetes mellitus & $1.076(0.498-2.325)$ & .853 & - & \\
\hline Hypertension & $2.058(0.879-4.819)$ & .107 & - & \\
\hline Dyslipidemia & $0.773(0.341-1.754)$ & .539 & - & \\
\hline History of smoking habits & $0.429(0.198-0.928)$ & .032 & $0.486(0.199-1.189)$ & .114 \\
\hline Beta-blockers & $3.318(0.448-24.548)$ & .24 & - & \\
\hline ACE-I or ARA & $1.000(0.235-4.259)$ & .998 & - & \\
\hline NYHA class & $0.871(0.419-1.807)$ & .71 & - & \\
\hline LBBB and $Q R S \geq 150 \mathrm{~ms}$ & $0.830(0.743-0.987)$ & .042 & $0.855(0.621-1.256)$ & .367 \\
\hline BNP & $1.001(1.000-1.001)$ & $<.001$ & $1.000(0.999-1.001)$ & .442 \\
\hline hs-CRP & $1.020(1.006-1.034)$ & .005 & $1.015(0.998-1.031)$ & .081 \\
\hline eGFR & $0.981(0.967-0.994)$ & .006 & $0.983(0.970-0.997)$ & .017 \\
\hline LVEF & $0.989(0.922-1.017)$ & .2 & - & \\
\hline LVEDV index & $1.005(0.996-1.015)$ & .255 & - & \\
\hline LVESV index & $1.004(0.993-1.015)$ & .517 & - & \\
\hline Early HMR & $0.614(0.016-1.654)$ & .125 & - & \\
\hline Late HMR & $0.034(0.003-0.420)$ & .008 & $0.066(0.005-0.880)$ & .04 \\
\hline Wash out rate & $1.019(0.994-1.044)$ & .14 & - & \\
\hline
\end{tabular}

$H R$, Hazards ratio; $C I$, confidence intervals; $N Y H A$, New York Heart Association; $A C E-I$, angiotensin-converting enzyme inhibitors; $A R A$, angiotensin II receptor blockers; $\angle B B B$, left bundle branch block; $B N P$, brain natriuretic peptide; $h s-C P R$, high-sensitivity Creactive protein; $e G F R$, estimated glomerular filtration rate; peak VO2, peak oxygen consumption; $L V E F$, left ventricular ejection fraction; $L V E D V$, LV end-diastolic volume; $L V E S V$, LV end-systolic volume; HMR, heart-to-mediastinum ratio

Table 3. Changes in clinical and functional parameter induced by CRT

\begin{tabular}{lllr}
\hline & \multicolumn{1}{c}{ Pre } & Post & P \\
\hline NYHA class $\geq$ III $(\%)$ & 73.8 & 13.5 & $<.001$ \\
BNP & $490( \pm 404)$ & $446( \pm 365)$ & .225 \\
hS-CRP & $3.2( \pm 2.8)$ & $3.7( \pm 1.5)$ & .292 \\
LVEF $(\%)$ & $24( \pm 7)$ & $37 \pm 11$ & .001 \\
LVEDV index & $127( \pm 40)$ & $106( \pm 43)$ & .01 \\
LVESV index & $95( \pm 36)$ & $71( \pm 37)$ & .001 \\
Early HMR & $1.47( \pm 0.17)$ & $1.49( \pm 0.17)$ & .456 \\
Late HMR & $1.38( \pm 0.18)$ & $1.41( \pm 0.19)$ & .159 \\
Wash out rate $(\%)$ & $49( \pm 18)$ & $47( \pm 16)$ & .602
\end{tabular}

$N Y H A$, New York Heart Association; $B N P$, brain natriuretic peptide; $h s-C P R$, high-sensitivity C-reactive protein; $L V E F$, left ventricular ejection fraction; $L V E D V$, LV end-diastolic volume; $L V E S V$, LV end-systolic volume; HMR, heart-to-mediastinum ratio

function and integrity of presynaptic nerve endings, since it uses the same myocardial uptake and release mechanisms as norepinephrine. ${ }^{14,15}$
Abnormal ${ }^{123}$ I-mIBG uptake and washout have been shown to predict HF progression and death. ${ }^{16}$ The ADMIRE-HF trial (961 patients, LVEF $\leq 35 \%$, and 
Table 4. Correlation between late HMR changes and modifications in clinical, laboratory, echocardiographic variables

\begin{tabular}{lcc}
\hline & \multicolumn{2}{c}{ Pearson correlation } \\
\cline { 2 - 3 } Dependent variable & $\boldsymbol{r}$ & $\boldsymbol{P}$ \\
\hline NYHA class change & -0.181 & .244 \\
BNP change & -0.265 & .103 \\
Hs-CRP change & -0.356 & .012 \\
eGFR change & -0.063 & .666 \\
Peak VO2 change & 0.547 & $<.001$ \\
LVEF change & 0.074 & .597 \\
LVEDV index change & 0.025 & .865 \\
LVESV index change & 0.032 & .825 \\
\hline
\end{tabular}

NYHA, New York Heart Association; BNP, brain natriuretic peptide; $h s-C P R$, high-sensitivity C-reactive protein; eGFR, estimated glomerular filtration rate; peak $\mathrm{VO}$, peak oxygen consumption; $L V E F$, left ventricular ejection fraction; $L V E D V$, LV end-diastolic volume; LVESV, LV end-systolic volume

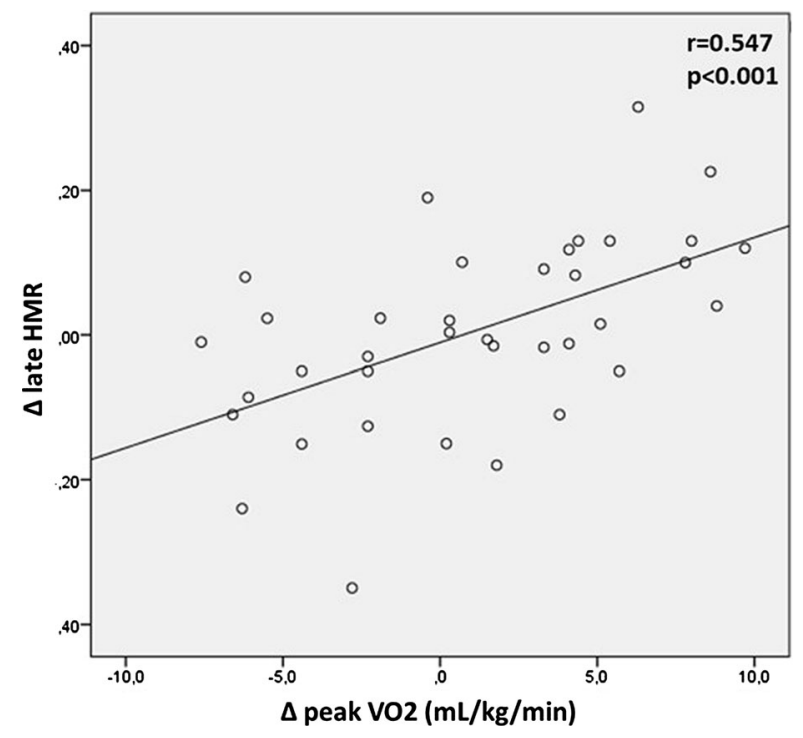

Figure 1. Relationship between changes induced by CRT in peak oxygen consumption (VO2) and late heart-to-mediastinum ratio (HMR) values.

NYHA class II-III) demonstrated that a higher late HMR was a strong independent predictor of prognosis. ${ }^{3}$ However, these data did not include patients after CRT implantation.

Some group reports have described an association between baseline ${ }^{123} \mathrm{I}$-mIBG parameters and response to CRT. Nishioka et $\mathrm{al}^{17}$ demonstrated that late HMR and washout rate were associated with CRT clinical response (improving to NYHA class I or II) in 30 chronic HF patients.

Cha et $\mathrm{al}^{18}$ demonstrated in 45 consecutive patients who received CRT (NYHA class II-III, mean LVEF $26 \pm 5 \%$ ) that baseline ${ }^{123} \mathrm{I}-\mathrm{mIBG}$ parameters were associated with a greater LV reverse remodeling.

Our study with 121 patients also demonstrated that baseline late HMR was an independent predictor of response to CRT at 6-month follow-up. Additionally, we also found that baseline late HMR was an independent predictor of composite outcome in HF patients who implant CRT.

These results are in line with Curcio et al study ${ }^{19}$ (26 patients, mean LVEF $29 \pm 4 \%$ ), in which late HMR showed a good performance for prediction of $\mathrm{HF}$ repeated hospitalizations.

These results suggest that baseline ${ }^{123} \mathrm{I}-\mathrm{mIBG}$ parameters may improve selection of patients to CRT implant.

\section{Cardiac Denervation Changes, CRT Response, and Functional Capacity Improvement}

Our results showed that, at 6-month after implantation, cardiac sympathetic denervation significantly improved in responders, whereas non-responders did not show significant changes.

Erol-Yilmaz et $\mathrm{al}^{20}$ studied 13 patients and also reported a significant improvement in ${ }^{123}$ I-mIBG parameters after 6-month follow-up, but the study did not analyze changes according to CRT response. Burri et $\mathrm{al}^{21}$ analyzed changes in ${ }^{123} \mathrm{I}-\mathrm{mIBG}$ parameters according to response, defined as an increase of $>5 \%$ in LVEF and improvement in NYHA class by $\geq 1$. In 8 of 13 patients who responded to CRT, washout rate significantly reduced and late HMR showed a statistically non-significant tendency to improve. In another study, Shinoahara et $\mathrm{al}^{22}$ defined response to CRT as a decrease in LVESV $\geq 15 \%$, and non-ischemic HF patients who were responders (19 patients) showed a significant increase in late HMR, whereas non-responders did not. Washout rate did not change in either group.

In this same study, ${ }^{22}$ improvement in cardiac sympathetic nervous dysfunction was associated with reduction in systemic inflammation. We also demonstrated an association between improvement in hs-CRP and late HMR modification. Moreover, there was a significant correlation between improvement in late HMR and improvement in peak VO2 because of CRT. This suggests that the alteration in cardiac adrenergic function may participate in the improvement in exercise tolerance conferred after CRT implantation. Results from previous studies $^{23-25}$ proposed that the correlation between 
increased sympathetic tone and exercise impairment is not restricted to sympathetic tone at the level of the peripheral muscle and the modification in cardiac sympathetic tone contributes to the observed changes in exercise tolerance. However, it is not possible to exclude that the explanation for the observed correlation between peak VO2 and late HMR may be because both parameters have been related to disease severity and prognosis ${ }^{26,27}$ and the relation between the two thus represents two different parameters of the same phenomenon.

\section{NEW KNOWLEDGE GAINED}

This study highlights the potential role of cardiac autonomic dysfunction assessed by ${ }^{123}$ I-mIBG imaging in CRT. Baseline late HMR was a predictor of response and clinical outcomes in HF patients undergoing CRT. Moreover, only CRT responders showed improvement in cardiac sympathetic nerve dysfunction and there was a significant correlation between changes in late HMR and modifications in peak VO2 after CRT.

\section{LIMITATIONS}

${ }^{123}$ I-mIBG scintigraphy results are highly dependent on acquisition and processing conditions, so this might preclude the extrapolation of this information to other settings, despite previous analysis of this population showed good to excellent reproducibility. ${ }^{8}$ Also, most of the subjects in the present study were treated with one or more drugs such as beta-blockers, angiotensin-converting enzyme inhibitors or angiotensin II receptor blockers which may influence myocardial uptake of ${ }^{123} \mathrm{I}$-mIBG. Another limitation may involve the fact that only planar images were used. Usually, the use of ${ }^{123} \mathrm{I}-\mathrm{mIBG}$ tomographic images allows a better separation of the heart from other thoracic structures. However, available data show that it is difficult to reconstruct SPECT data when global ${ }^{123}$ I-mIBG uptake is severely reduced, like in severely dilated cardiomyopathy. ${ }^{28}$ Finally, the limited sample size makes analysis of the study less robust.

\section{CONCLUSION}

Baseline cardiac sympathetic nervous dysfunction assessed by ${ }^{123} \mathrm{I}$-mIBG imaging predicted CRT response and outcomes in advanced HF patients. Cardiac autonomic positive modulation occurred only in patients who responded to CRT with significant reverse remodeling. This autonomic change correlated with objective increase in functional capacity.

These results may help deciding whose patients will respond to CRT in cases of doubt regarding decision of implantation.

\section{Disclosures}

Rita Ilhão Moreira, Ana Abreu, Guilherme Portugal, Luís Oliveira, Mário Oliveira, Inês Rodrigues, Madalena Coutinho Cruz, Pedro Silva Cunha, Vanessa Santos, Helena Santa Clara, Rui Cruz Ferreira, Miguel Mota Carmo have no conflicts of interest to declare.

\section{References}

1. Leimbach WN, Wallin BG, Victor RG, Aylward PE, Sundlöf G, Mark AL. Direct evidence from intraneural recordings for increased central sympathetic outflow in patients with heart failure. Circulation 1986;73:913-9.

2. Packer M. The neurohormonal hypothesis: A theory to explain the mechanism of disease progression in heart failure. J Am Coll Cardiol 1992;20:248-54.

3. Jacobson AF, Senior R, Cerqueira MD, Wong ND, Thomas GS, Lopez VA, et al. Myocardial iodine-123 meta-iodobenzylguanidine imaging and cardiac events in heart failure. Results of the prospective ADMIRE-HF (AdreView Myocardial Imaging for Risk Evaluation in Heart Failure) study. J Am Coll Cardiol 2010;55:2212-21.

4. Anastasiou-Nana MI, Terrovitis JV, Athanasoulis T, Karaloizos L, Geramoutsos A, Pappa L, et al. Prognostic value of iodine-123metaiodobenzylguanidine myocardial uptake and heart rate variability in chronic congestive heart failure secondary to ischemic or idiopathic dilated cardiomyopathy. Am J Cardiol 2005;96:427-31.

5. Bristow MR, Saxon LA, Boehmer J, Krueger S, Kass DA, De Marco T, et al. Cardiac-resynchronization therapy with or without an implantable defibrillator in advanced chronic heart failure. $\mathrm{N}$ Engl J Med 2004;350:2140-50.

6. Cleland JG, Daubert JC, Erdmann E, Freemantle N, Gras D, Kappenberger L, et al. The effect of cardiac resynchronization on morbidity and mortality in heart failure. $\mathrm{N}$ Engl $\mathrm{J}$ Med 2005;352:1539-49.

7. Tang ASL, Wells GA, Talajic M, Arnold MO, Sheldon R, Connolly $\mathrm{S}$, et al. Cardiac-resynchronization therapy for mild-tomoderate heart failure. N Engl J Med 2010;363:2385-95.

8. Cruz MC, Abreu A, Portugal G, Santa-Clara H, Cunha PS, Oliveira MM, et al. Relationship of left ventricular global longitudinal strain with cardiac autonomic denervation as assessed by ${ }^{123} \mathrm{I}-\mathrm{mIBG}$ scintigraphy in patients with heart failure with reduced ejection fraction submitted to cardiac resynchronization therapy: Assessment of cardiac autonomic denervation by GLS in patients with heart failure with reduced ejection fraction submitted to CRT. J Nucl Cardiol 2017. https://doi.org/10.1007/s12350-0171148-9.

9. Lang RM, Badano LP, Mor-Avi V, Afilalo J, Armstrong A, Ernande L, et al. Recommendations for cardiac chamber quantification by echocardiography in adults: An update from the American society of echocardiography and the European association of cardiovascular imaging. Eur Heart J Cardiovasc Imaging 2015;16:233-71.

10. Nagueh SF, Smiseth OA, Appleton CP, Byrd BF, Dokainish H, Edvardsen $\mathrm{T}$, et al. Recommendations for the evaluation of left ventricular diastolic function by echocardiography: An update from the American Society of Echocardiography and the European Association of Cardiovascular Imaging. J Am Soc Echocardiogr 2016;29:277-314

11. Boidol J, Średniawa B, Kowalski O, Szulik M, Mazurek M, Sokal A, et al. Many response criteria are poor predictors of outcomes 
after cardiac resynchronization therapy: Validation using data from the randomized trial. Europace 2013;15:835-44.

12. Zucker IH, Wang W, Pliquett RU, Liu JL, Patel KP. The regulation of sympathetic outflow in heart failure. The roles of angiotensin II, nitric oxide, and exercise training. Ann N Y Acad Sci 2001;940:431-43.

13. Kaye DM, Lefkovits J, Jennings GL, Bergin P, Broughton A, Esler MD. Adverse consequences of high sympathetic nervous activity in the failing human heart. J Am Coll Cardiol 1995;26:1257-63.

14. Schofer J, Spielmann R, Schuchert A, Weber K, Schlüter M. Iodine-123 meta-iodobenzylguanidine scintigraphy: A noninvasive method to demonstrate myocardial adrenergic nervous system disintegrity in patients with idiopathic dilated cardiomyopathy. J Am Coll Cardiol 1988;12:1252-8.

15. Imamura Y, Ando H, Mitsuoka W, Egashira S, Masaki H, Ashihara $\mathrm{T}$, et al. Iodine-123 metaiodobenzylguanidine images reflect intense myocardial adrenergic nervous activity in congestive heart failure independent of underlying cause. J Am Coll Cardiol 1995;26:1594-9.

16. Verberne HJ, Brewster LM, Somsen GA, Van Eck-Smit BLF. Prognostic value of myocardial ${ }^{123}$ I-metaiodobenzylguanidine (MIBG) parameters in patients with heart failure: A systematic review. Eur Heart J 2008;29:1147-59.

17. Nishioka SADO, Martinelli M, Brandão SCS, Giorgi MC, Vieira MLC, Costa R, et al. Cardiac sympathetic activity pre and post resynchronization therapy evaluated by 123I-MIBG myocardial scintigraphy. J Nucl Cardiol 2007;14:852-9.

18. Cha Y-M, Chareonthaitawee P, Dong YX, Kemp BJ, Oh JK, Miyazaki $\mathrm{C}$, et al. Cardiac sympathetic reserve and response to cardiac resynchronization therapy. Circ Heart Fail 2011;4:339-44.

19. Curcio A, Cascini GL, De Rosa S, Pasceri E, Veneziano C, Cipullo $\mathrm{S}$, et al. 123I-mIBG imaging predicts functional improvement and clinical outcome in patients with heart failure and CRT implantation. Int J Cardiol 2016;207:107-9.

20. Erol-Yilmaz A, Verberne HJ, Schrama TA, Hrudova J, De Winter RJ, Van Eck-Smit BLF, et al. Cardiac resynchronization induces favorable neurohumoral changes. Pacing Clin Electrophysiol 2005;28:304-10.

21. Burri H, Sunthorn H, Somsen A, Fleury E, Stettler C, Shah D, et al. Improvement in cardiac sympathetic nerve activity in responders to resynchronization therapy. Europace 2008;10:374-8.

22. Shinohara T, Takahashi N, Saito S, Okada N, Wakisaka O, Yufu $\mathrm{K}$, et al. Effect of cardiac resynchronization therapy on cardiac sympathetic nervous dysfunction and serum C-reactive protein level. Pacing Clin Electrophysiol 2011;34:1225-30.

23. Cohen-Solal A, Esanu Y, Logeart D, Pessione F, Dubois C, Dreyfus $\mathrm{G}$, et al. Cardiac metaiodobenzylguanidine uptake in patients with moderate chronic heart failure: Relationship with peak oxygen uptake and prognosis. J Am Coll Cardiol 1999;33:759-66.

24. Atsumi H, Takeishi Y, Fujiwara S, Tomoike H. Cardiac sympathetic nervous disintegrity is related to exercise intolerance in patients with chronic heart failure. Nucl Med Commun 1998;19:451-6.

25. De Milliano PAR, Van Eck-Smit BLF, Van Zwieten PA, De Groot AC, Tijssen JGP, Lie KI. Relationship between cardiac metaiodobenzylguanidine uptake and hemodynamic, functional and neurohormonal parameters in patients with heart failure. Eur $\mathbf{J}$ Heart Fail 2001;3:693-7.

26. Nakata T, Nakajima K, Yamashina S, Yamada T, Momose M, Kasama S, et al. A pooled analysis of multicenter cohort studies of 123I-mIBG imaging of sympathetic innervation for assessment of long-term prognosis in heart failure. JACC Cardiovasc Imaging 2013;6:772-84.

27. Alba AC, Adamson MW, MacIsaac J, Lalonde SD, Chan WS, Delgado $\mathrm{DH}$, et al. The added value of exercise variables in heart failure prognosis. J Card Fail 2016;22:492-7.

28. Stefanelli A, Treglia G, Giordano A. (123)I-MIBG scintigraphy as a powerful tool to plan an implantable cardioverter defibrillator and to assess cardiac resynchronization therapy in heart failure patients. Int J Mol Imaging. 2012;2012:690468. 